\title{
Prognostic value of atrial fibrillation in heart failure with preserved ejection fraction at mid-term follow-up
}

\author{
Katarina Cenkerova ${ }^{1 *}$, Juraj Dúbrava ${ }^{2}$, Veronika Pokorna² ${ }^{2}$ Jozef Kalužay ${ }^{2}$, Olga Jurkovičováa \\ ${ }^{1}$ The National Institute of Cardiovascular Disease, Bratislava, Slovak Republic \\ ${ }^{2}$ University Teaching Hospital of St. Cyril and Methodius, Bratislava, Slovak Republic
}

\begin{abstract}
Aim: To assess the influence of atrial fibrillation on mortality in heart failure with preserved ejection fraction (HFPEF) in a prospective study compared to heart failure with reduced ejection fraction (HFREF). We have hypothesized that atrial fibrillation decreases survival in HFPEF.

Patients and Methods: The study included a total of 109 patients admitted to Medical wards for heart failure within one year's period (2010-2011). The follow-up was 24 months. Patients were divided into two groups based on left ventricular ejection fraction (LVEF); HFPEF with LVEF more than $40 \%(n=64)$ and HFREF with LVEF less than $40 \%(n=45)$. For each patient we evaluated the presence of atrial fibrillation (AF) on ECG in the history and on admission. Data were analyzed using JMP9 statistical program. Unless otherwise specified, the data are presented as means.
\end{abstract}

\section{Received: $18^{\text {th }}$ Apr 2014}

*Address for correspondence: Department of General Cardiology, The National Institute of Cardiovascular Disease, Pod Krásnou hôrkou 1, 833 48, Bratislava, Slovak Republic

Phone: +421-905972249

E-mail: kmardiakova@gmail.com
Results: The prevalence of history of AF was significantly higher in HFPEF vs. HFREF ( $67 \%$ vs. $44 \%, p<0.05$ ). We observed a trend of higher prevalence of $A F$ on admission in HFPEF vs. HFREF ( $50 \%$ vs. $29 \%, p=0.058)$ and significantly higher prevalence of non-sinus rhythm on admission in HFPEF vs. HFREF ( $56 \%$ vs. $34 \%, p<0.05)$. There was no significant difference in hospital mortality, cardiovascular mortality and all-cause mortality among patients with and without the history of AF neither in HFPEF nor in HFREF. The same results were found when comparing patients with and without the presence of $\mathrm{AF}$ on admission. AF was not an independent predictor of mortality.

Conclusion: We observed significantly higher prevalence of history of AF and significantly higher prevalence of nonsinus rhythm on admission in HFPEF vs. HFREF. We found a trend of higher prevalence of AF on admission in HFPEF vs. HFREF. We did not find AF to be a predictor of two-year mortality neither in patients with HFPEF nor in patients with HFREF.

KEYWORDS: heart failure with preserved ejection fraction, atrial fibrillation, prognosis.

CITATION: Cardiol Croat. 2014;9(5-6):230. 